\title{
Ligament Formation and Breakup in Two-Fluid Spray Atomization
}

\author{
Isaac M. Jackiw*, Nasser Ashgriz \\ Department of Mechanical and Industrial Engineering, University of Toronto, Toronto, Canada \\ ${ }^{*}$ Corresponding author email: isaac.jackiw@mail.utoronto.ca
}

\begin{abstract}
The present work studies low-viscosity two-fluid atomization experimentally and analytically in order to characterize and predict the formation and breakup of ligaments in the spray. The study is based on experiments conducted using commercially available two-fluid nozzles with water as the liquid. Shadowgraph images were used to visualize and characterize the nearnozzle flow and ligaments while the droplet size distribution was measured in the far-field using a Malvern Spraytec. Our images reveal that two different wave structures contribute to the breakup: surface waves, and bulk waves. These waves eventually result in the formation of structures that breakup with a mechanism similar to droplet breakup, where small droplets are formed from the surface waves and large droplets form from the bulk waves. A recent deformation-rate based droplet breakup model is applied to the surface and bulk wave geometries to predict the formation and breakup of ligaments. These predictions are compared to measurements of the ligament sizes from the shadowgraph images as well as the droplet size distributions with good agreement in the order of magnitude and trends of each mode.
\end{abstract}

\section{Keywords}

Atomization; Experiment; Modelling; Primary breakup; Twin-fluid nozzle

\section{Introduction}

Spray atomization has a wide variety of applications ranging from the manufacturing of pharmaceuticals to the production of metal powders for use in additive manufacturing. Despite the prolific and long-running use of sprays in industry, the understanding of the atomization process is not yet well enough understood to attain good prediction of the sizes of the drops produced by the spray when varying the working fluids or the nozzle design and scale [1]. Analytical modelling of spray atomization aims to provide prediction of the spray droplet sizes by reducing the problem to the underlying physical mechanisms of the breakup, and has several key advantages over empirical and numerical simulation strategies such as providing an intuitive understanding of the spray behaviour, as well as facilitating the design and real-time optimization of processes involving new fluids.

The predominant analytical models in the literature for two-fluid atomization break the process up into multiple steps. First, the Kelvin-Helmholtz shear instability is presumed to form waves on the liquid jet surface, which are drawn out by the air-stream into 'liquid tongues'. These liquid tongues are then accelerated by the airflow and undergo breakup by the Rayleigh-Taylor instability. This process was described and modelled for inviscid breakup [2] and for viscous non-Newtonian breakup [3], implementing the work of Joseph et al for inviscid [4] and viscid droplet breakup [5], respectively, in order to model the breakup of the liquid tongues by the Rayleigh-Taylor instability as 'droplet-analogues'. However, this description has several shortcomings that stem from the description not being a physically-accurate representation of the process at scales relevant to industry.

Firstly, the breakup is presumed to occur solely on the liquid tongues that develop from surface waves on the liquid jet. While these tongues have been observed in previous works [6], they only form at relatively large scales and low gas flow speeds. In practical cases, the initial symmetric waves form pairs that lead to sinuous waves in the liquid jet bulk [7], which break when they waver into the air-stream. 
Secondly, the Rayleigh-Taylor droplet breakup description is only applicable to the catastrophic breakup morphology and does not account for the other morphologies that occur in two-fluid atomization such as bag and membrane breakup, which occur more frequently in high-viscosity atomization where the air flows required to achieve catastrophic atomization may be unattainable. As a result, the Rayleigh-Taylor breakup mechanism does not model or predict the formation of ligaments and membranes, which are important structures observed in high-viscosity atomization. Finally, these approaches are insufficient in describing the multi-modal droplet size distributions that have been reported in other works [8]. In order to develop improved analytical models for two-fluid atomization, these deficiencies must be addressed.

Recently, the present authors proposed a new analytical framework for droplet breakup, which postulates that the rate of deformation of the droplet governs how it breaks [9]. The model developed in this work was shown to provide good prediction of the formation and breakup of ligaments from droplet breakup for several morphologies, offering a significant improvement over previous Rayleigh-Taylor instability based models.

The droplet breakup model starts by predicting the radial deformation rate of the drop, $\dot{d}$, based on the Weber number, $W e$, and deformation time constant, $\tau$, of the drop (equation 1),

$$
\frac{\dot{d}}{d_{0}}=\frac{1.125}{\tau}\left(1-\frac{32}{9 W e}\right)
$$

The deformation rate is then used to predict the initial thickness of the ligament formed by the rim at the periphery of the droplet, $d_{i n i}$ (equation 2),

$$
\frac{d_{i n i}}{d_{0}}=\frac{4}{\left(\rho_{l} \dot{d}^{2} d_{0} / 4 \sigma+10.4\right)}
$$

Following the expansion of rim due to the formation of bags, the ligament thins to its final diameter, $d_{f i n}$, and breaks by the Rayleigh-Plateau capillary instability to give the child drop size from the breakup of the rim, $d_{c}$ (equation 3). Note that, for the purposes of this paper, all constants have been substituted into the equations, and an empirical relation for the rim expansion sub-process has been used for simplicity.

$$
\frac{d_{c}}{d_{0}}=1.89 \frac{d_{i n i}}{d_{0}} \sqrt{\frac{d_{i n i}}{d_{f i n}}}, \quad \frac{d_{i n i}}{d_{f i n}} \approx \frac{1}{0.64}
$$

The present study seeks to apply this breakup modelling approach in the framework of two-fluid sprays to provide prediction of the formation and breakup of ligaments.

\section{Material and methods}

The nozzle used in this study was an externally mixing two-fluid nozzle having a liquid orifice diameter of $d_{l}=0.71 \mathrm{~mm}$ and annular gas inside and outside orifice diameters of $d_{g, i}=1.6 \mathrm{~mm}$ and $d_{g, o}=1.78 \mathrm{~mm}$, respectively (Spraying System Co. 1/4J Series set-up SU2). Water was used as the atomized fluid ( $\rho_{l}=1000 \mathrm{~kg} / \mathrm{m}^{3}, \mu_{l}=1 \mathrm{mPa} . \mathrm{s}, \sigma=0.0729 \mathrm{~N} / \mathrm{m}$ ) and was fed to the nozzle from a pressurised reservoir metered by a rotameter (OMEGA FLDW3309ST). Air was used as the atomizing gas and was supplied to the nozzle by the building's compressed air system. The air flow-rate was measured using a rotometer (Cole Parmer 03217-34) and a pressure transducer (WIKA A-10). Due to the considerable challenges of directly measuring very small, high-speed, compressible gas jet flows that issue from the nozzle, the properties of the gas flow in the present study are calculated assuming isentropic compressible flow through the nozzle with a gas specific heat ratio of $k=1.4$, specific gas constant of $R_{*}=287 \mathrm{~J} / \mathrm{kg} . \mathrm{K}$, and upstream stagnation temperature of $20^{\circ} \mathrm{C}$. The isentropic mass flow rate, $\dot{m}_{g, i s e n}$, is adjusted 
using the nozzle discharge coefficient, $C_{d}$, determined empirically as $C_{d}=0.83$, to match the measured mass flow rate as $\dot{m}_{g}=C_{d} \dot{m}_{g, i s e n}$, and to account for the boundary layer effects in the very small gas orifice that are neglected in the isentropic flow assumption. This correction was also used to adjust the gas speed, $u_{g}$, assuming that the the boundary layer effects do not affect the gas density, $\rho_{g}$, significantly. Although water is of relatively low viscosity, tests were performed at flow conditions which result in bag and membrane breakup morphologies commonly seen in high-viscosity atomization as well as at the flow conditions recommended by the nozzle manufacturer for water.

Measurement of the droplet or particle size distribution (PSD) was carried out using a Malvern Spraytec Particle sizer with a $300 \mathrm{~mm}$ lens. The measurement beam was centred on the spray at a distance $20 \mathrm{~mm}$ downstream of the nozzle exit in order to measure the sizes resulting predominantly from the primary atomization before further breakup occurs. An extraction system was used to prevent spray accumulation inside the chamber that would interfere with the imaging and measurement devices.

Shadowgraph imaging of the near nozzle region was carried out using a Mazlite Dropsizer with a resolution of $0.0024 \mathrm{~mm} /$ pixel, a sensor size of $3088 \times 2076$ pixels, and 1x magnification giving a field of view of $7.41 \times 4.98 \mathrm{~mm}$. 200 images were taken at each flow condition. Several factors impede the accurate quantitative measurement of spray images. Due to the narrow depth of field of such imaging devices, many of the structures are somewhat out of focus, impeding the clear identification of their edges. Additionally, the dynamic growth and thinning of the ligaments makes defining $d_{i n i}$ and $d_{f}$ from still images impossible. Owing to the corrugation of the ligaments, there is also no single size that is characteristic of all ligaments in the spray. Furthermore, the complexity of the ligament networks due to turbulence and advection of the liquid jet makes automated measurement very difficult. To make manual measurement if the images tractable, one measurement was taken per image of the ligament thickness for each type of breakup where the structures could be clearly identified using ImageJ. The reported values in this study are the average over all images for each condition, with error bars showing the standard deviation of the measurement set. As a result, the ligament measurements reported in the present work should not be considered definitive, and are used only as a means of verifying and assessing the intermediate steps of the model in a semi-quantitative manner. Despite these shortcomings, this is the first work to the authors' knowledge to show any form of quantitative ligament size measurement in two-fluid nozzle atomization.

\section{Results and discussion}

As described in the introduction, two types of waves are present in practical two-fluid sprays: varicose surface waves, and sinuous bulk waves. The near-nozzle images of the present study, for example Figure $1(a)$, show that both types of waves are susceptible to breakup by the airflow.

When the bulk waves break, they introduce a characteristic size proportional to the liquid jet diameter. By contrast, the surface waves present a smaller characteristic size, as only a portion of the liquid jet is exposed. As a result, the surface waves break as relatively thinner ligaments than the bulk waves, which we suggest to be one of the causes of the multi-modal distributions sometimes observed in two-fluid sprays [8]. Therefore, it is necessary to define the characteristic size of both surface and bulk waves.

Following the concept of the droplet breakup analogue, the surface waves are presumed to grow until the 'droplet' contained in the surface wave becomes large enough to be susceptible to the breakup, illustrated in Figure $1(b)$. The characteristic size of the surface wave, $d_{s}$, is assumed to be the amplitude of the surface wave when it becomes susceptible to this breakup. The bulk waves break when they waver into the gas flow, therefore, as a simplification, the geometry of the breakup is assumed to take the form of a cylinder that is exposed to the gas flow perpendicularly to its axis, illustrated in Figure $1(c)$. In this case, the characteristic size of the bulk wave, $d_{b}$, is assumed to be the diameter of the liquid jet. Note that although the 


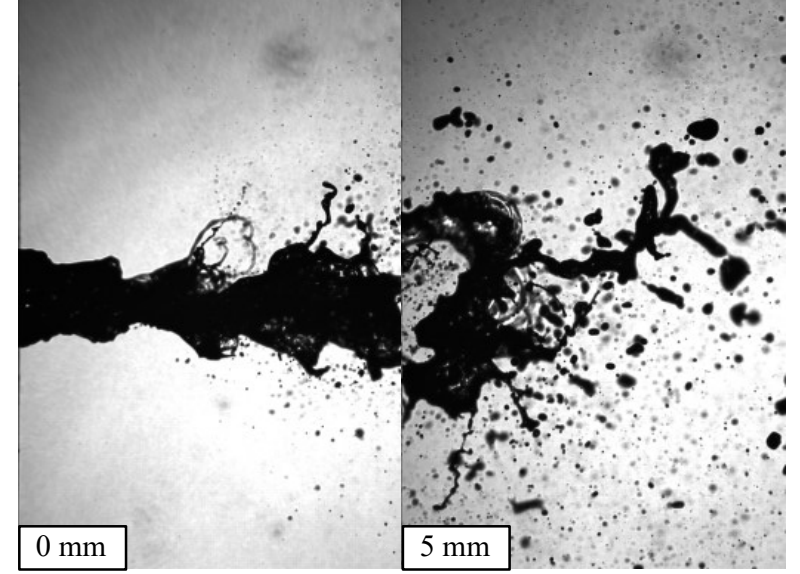

(a) Image of spray

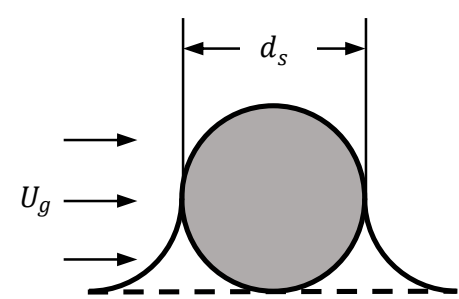

(b) Surface wave breakup geometry

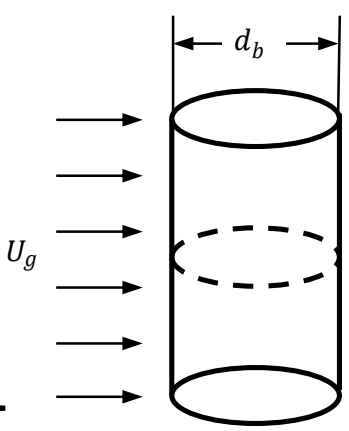

(c) Bulk breakup geometry

Figure 1. (a) Images of spray showing transition from varicose to sinuous waves and the surface and bulk breakup mechanisms, with illustrations showing the $(b)$ surface wave and $(c)$ core breakup geometries.

geometry in this case differs from the droplet model being applied, previous works have shown that the aerodynamic breakup of cylindrical columns is similar to that of droplets [10]. Other analytical works have also applied droplet-based models to the breakup of liquid columns, such as the liquid-tongues described in the introduction.

The second parameter required by the model is the characteristic speed, which is assumed to be the relative velocity between the liquid and gas flows, $u_{r}=u_{g}-u_{l}$; however, since $u_{g}>>u_{l}$ in two-fluid nozzles, $u_{r} \approx u_{g}$.

In the following sections, the model of [9] described in the introduction will be applied to both the bulk and surface wave geometries. Since the bulk breakup geometry is larger than that of the surface waves, the bulk breakup mechanism is expected to dominate the spray behaviour. Therefore, bulk wave breakup will be discussed first.

\section{Bulk wave breakup}

Bulk breakup occurs when the sinuous bulk waves cross into the air stream, introducing the cylindrical bulk geometry of characteristic size $d_{b}$, depicted in Figure $1(b)$, to the air-flow. Although previous models, analytical and empirical, consider the liquid jet to be of the same diameter as the liquid orifice, $d_{l}$, in practical nozzles the wall of the nozzle separating the liquid and gas flows is of a finite thickness proportional to $d_{l}$. Thus, the liquid jet wets across the face of the nozzle and takes on the diameter of the annular gas jet inner diameter, $d_{g, i}$. This phenomenon was first observed by [11] and is consistent with our images of the nozzle flow in the present work. As such, $d_{g, i}$ will be used as a first approximation to the characteristic diameter in the bulk breakup model, neglecting the thinning of the liquid stream due to advection by the gas flow or mass-loss from prior breakup of the surface waves.

To verify the ligament formation theory of the model, the measured representative ligament sizes are compared to the expected range given by the upper $\left(d_{i n i}\right)$ and lower $\left(d_{f i n}\right)$ limits of the theory from equation 2 and $d_{\text {ini }} / d_{\text {fin }} \approx 1 / 0.64$ in Figure $2(a)$.

Reasonable agreement is found in the order of magnitude of the ligament thickness as well as with the trend of decreasing ligament thickness with increasing $\dot{m}_{g}$; however, it is clear that the prediction is somewhat low. This may be the result of the theory not yet accounting for the effects of increasing $\dot{m}_{l}$ on the characteristic diameter and velocity. It is worth nothing, however, that changes to $u_{l}$ only very weakly affect $u_{r}$, and that the variation in liquid flow rate shown in the experiments is of the same order as the standard deviation in the measurements. Another possible source of this error may be the relatively large measurement error as a result of ligament edges being somewhat out-of-focus, which would bias the measurements to be somewhat larger.

Since the breakup of the bulk waves is expected to dominate the SMD as it accounts for the 


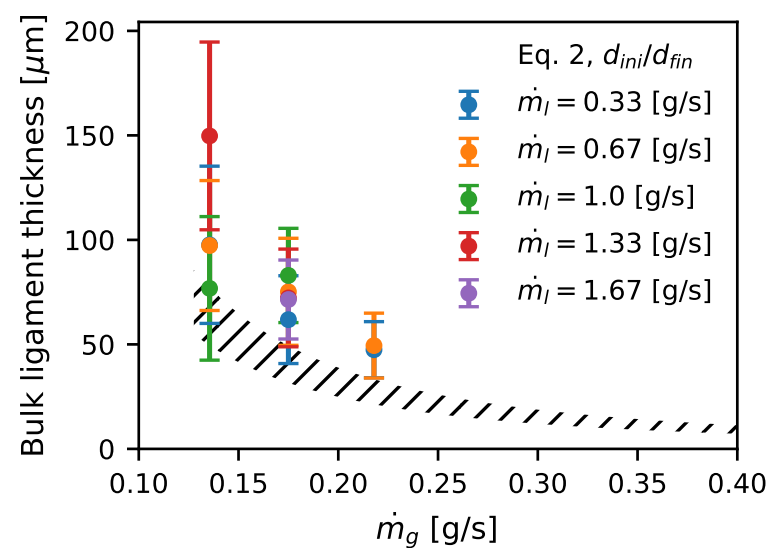

(a)

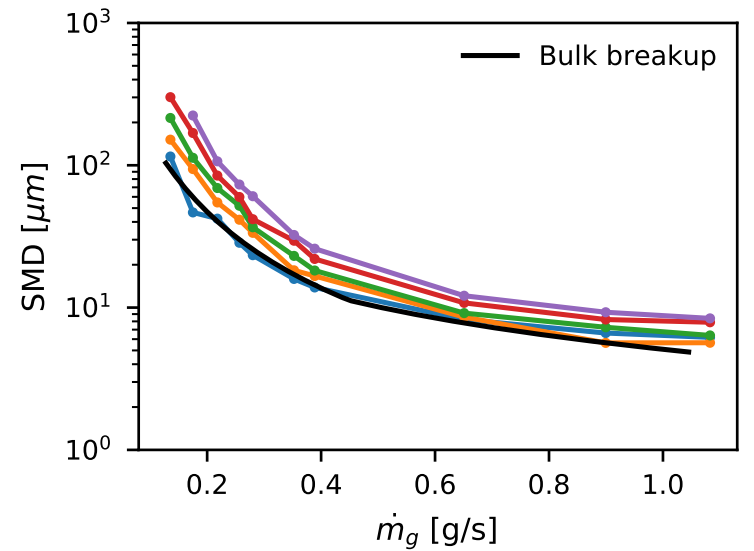

(b)

Figure 2. (a) Comparison of bulk ligament thickness theory (eq. 2 and $d_{\text {ini }} / d_{\text {fin }} \approx 1 / 0.64$ ) to representative bulk ligament measurements. (b) Comparison of bulk breakup model to SMD measurements. Experiments shown for varying $\dot{m}_{l}$ and $\dot{m}_{g}$.

breakup of the largest characteristic size, and thus \% volume, of the spray, the bulk ligament breakup size prediction of equation 3 is compared to the measured SMD in Figure $2(b)$. Excellent agreement between the predicted bulk breakup size and the measured SMD for varying $\dot{m}_{g}$ is found, indicating that the primary aerodynamic effects are well represented by the model. However, the trends of increasing SMD with increasing $\dot{m}_{l}$ are not captured. The effect of increasing liquid flow becomes clear when observing the trends in PSD shown in Figure 3.

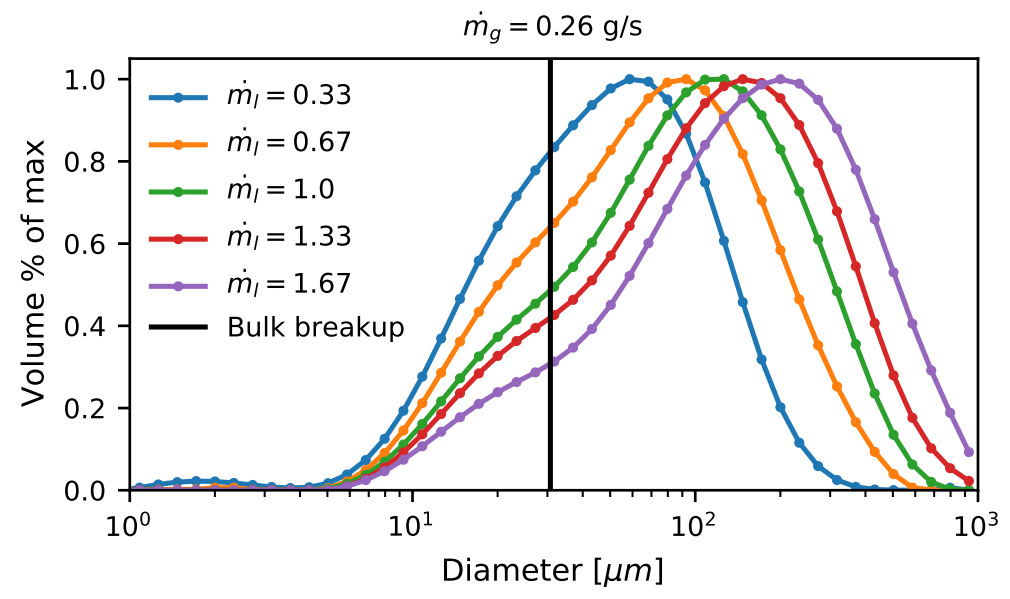

Figure 3. Comparison of Bulk breakup theory to PSD measurements for varying $\dot{m}_{l}$ at $\dot{m}_{g}=0.26 \mathrm{~g} / \mathrm{s}$.

Figure 3 shows that there are large droplets on the order of $100 \mu \mathrm{m}$ present at high liquid flow rates. These sizes lead to a bimodal distribution where a large mode, which is more strongly affected by $\dot{m}_{l}$, is dominant at higher $\dot{m}_{l}$. These large sizes are on the order of $d_{b}$, and thus are not the direct result of aerodynamic breakup.

The near-nozzle images show evidence of such large drops being formed when only a portion of a bulk wave breaks. This suggests that the bulk breakup occurs intermittently between the waves due to the sinuous shape of the liquid jet, causing fragments to be split-off between the bulk breakup events, forming large droplets on the order of $d_{b}$. This is effectively an indicator of incomplete atomization, where the mass flow of the liquid jet exceeds the rate of mass removal from the liquid jet via aerodynamic breakup, which explains why this size and its frequency increase with $\dot{m}_{l}$ and decreases with $\dot{m}_{g}$ as will be shown in the next section. 


\section{Surface wave breakup}

In surface breakup, the surface waves grow as a result of exposure to the air stream via a Kelvin-Helmholtz shear instability. Following with the droplet-breakup analogy, it is suggested that these surface waves will be susceptible to breakup when they reach the size that satisfies the minimum $W e$ required for breakup, $W e=8.8$ [9], which defines the characteristic size for surface breakup, $d_{s}=W e \sigma / \rho u_{r}^{2}$. Since the surface waves will always break at this condition, the expected breakup morphology is bag breakup. The exceptions for this are the limiting cases of very low and very high gas flow rates. At very low flow rates and for a sufficiently large $d_{l}$, the wave crest can grow to the extent that it digitates to form a liquid tongue, as described by [6], and never attains $W e=8.8$. This limit does not occur in practical two-fluid nozzles as the scales are much smaller such $d_{s}$ is always below the capillary length at which this mechanism occurs. At very high gas speeds, the gas flow may be able to shear the wave from the surface as in the Shear-Thinning breakup morphology of droplets, which was also modelled in [9], although this is difficult to verify by imaging due to the very small time and spacial scales of the phenomenon. As before, the measured representative ligament sizes are compared to the expected range given by the upper and lower limits of the theory using $d_{s}$ as the characteristic size, as shown in Figure $4(a)$.

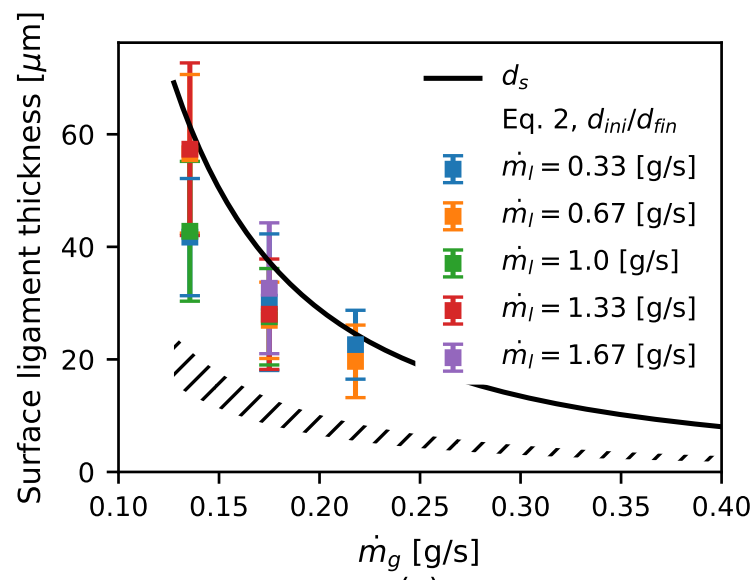

(a)

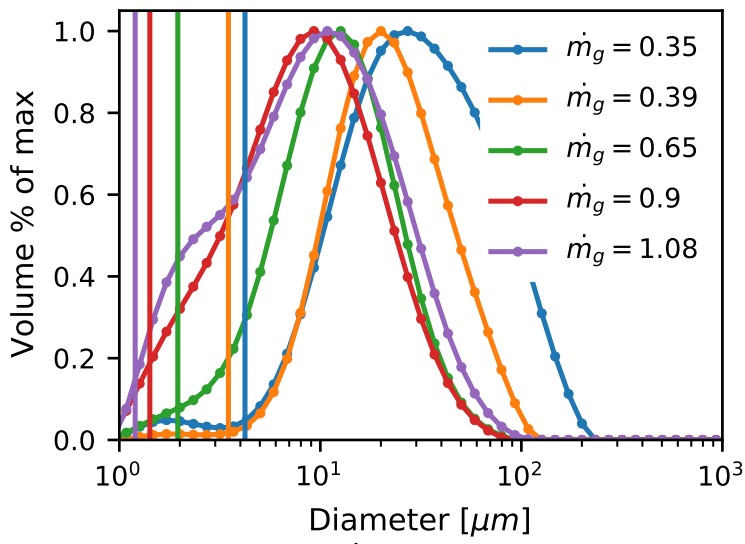

(b) $\dot{m}_{l}=0.67[\mathrm{~g} / \mathrm{s}]$

Figure 4. (a) Comparison of $d_{s}$ and surface ligament thickness theory (eq. 2 and $d_{\text {ini }} / d_{\text {fin }} \approx 1 / 0.64$ ) to representative surface ligament measurements for varying $\dot{m}_{l}$ and $\dot{m}_{g} .(b)$ Comparison of surface breakup model (solid vertical lines) to PSD measurements at fixed $\dot{m}_{l}=0.67 \mathrm{~g} / \mathrm{s}$ and varying $\dot{m}_{g}$.

Figure $4(a)$ shows that the measured surface ligament sizes are clearly larger than the theory predicts. This is because the theory at present does not include viscous effects, which become important in droplet breakup when $O h>0.01$ [12]. For the present experiments using water, this occurs when $d_{s}<100 \mu \mathrm{m}$, which occurs for all cases of surface breakup as shown in Figure $4(a)$. Including viscosity in the analysis will increase the minimum $W e$ required for breakup, and will affect the model by decreasing the deformation rate (equation 1), which results in a larger ligament size for a given $W e$.

Since the surface breakup sizes are very small, surface breakup does not significantly contribute to the PSD or SMD unless it occurs very frequently. This occurs at high $\dot{m}_{g}$ where, owing to a shorter wavelength, more surface waves are present on the liquid jet and more instances of breakup occur around each wave. Figure $4(b)$ compares the surface breakup size prediction to the measured PSD for varying $\dot{m}_{g}$ at a fixed $\dot{m}_{l}$. As $\dot{m}_{g}$ increases, the largest mode generated by the intermittent breakup of the bulk disappears as more of the liquid jet is removed by the surface breakup mechanism allowing for a more complete atomization for the given $\dot{m}_{l}$. For a relatively narrow range of operation (see $\dot{m}_{g}=0.39$ in Figure $4(b)$ ), only the bulk breakup mechanism dominates, and the spray is essentially mono-modal. However, as $\dot{m}_{g}$ increases further, an additional small mode $O(1 \mu \mathrm{m})$ appears, which is in agreement with 
the magnitude predicted by the surface breakup mechanism (indicated for each flow condition by solid vertical lines).

This result provides the interesting conclusion that a bimodal distribution does not only occur for cases of incomplete atomization, but also for cases where $\dot{m}_{g}$ is sufficiently high to cause the surface breakup mechanism to become significant compared the the bulk breakup mechanism. This understanding therefore suggests both a lower and upper limit of $\dot{m}_{g}$ for attaining a mono-modal distribution from a two-fluid spray. Further development of the model to include a prediction of the breakup frequency of each mode will likely result in the quantification of these limits.

\section{Conclusions}

In the present work, low-viscosity atomization was studied in order to characterize and predict the formation and breakup of ligaments in the spray, resulting in the following conclusions:

1. Multiple modes of breakup contribute to the atomization two-fluid nozzle sprays leading to multi-modal PSDs; surface, bulk, and intermittent bulk breakup modes.

2. Increasing $\dot{m}_{l}$ primarily increases the size and volume frequency of the intermittent bulk breakup mode, which can lead to a bimodal distribution.

3. Increasing $\dot{m}_{g}$ decreases the breakup sizes of each mode while simultaneously decreasing the frequency of intermittent bulk breakup and increasing the frequency of surface breakup. This results in a narrow range of $\dot{m}_{g}$ where a mono-modal distribution dominated by the bulk breakup mode can be achieved.

4. The authors' recent deformation-rate based droplet breakup model [9] shows good agreement with SMD and PSD measurements for increasing $\dot{m}_{g}$ when used to model the surface and bulk breakup modes.

5. Ligament thickness measurements were reported for the first time and were used to verify the intermediate ligament formation steps of the model for both surface and bulk breakup modes with reasonable agreement for the bulk breakup mode.

6. Owing to the very small size of the surface breakup geometry, the effect of viscosity on this mode cannot be neglected, even for a relatively inviscid fluid such as water.

\section{Future work}

The further development of this work will seek to model and understand the effect of the flow conditions and nozzle geometry on the relative frequency of each mode in an effort to provide a more complete prediction of the PSD.

\section{Acknowledgements}

The authors acknowledge the financial support of the International Fine Particle Research Institute (IFPRI).

The authors also acknowledge the 2019-2020 MUSSL capstone team; Nasa Chau Nguyen, Rucheng Wang, Ziyu Wang, and Li Zhu; for helping with the acquisition of the PSD data. 


\section{Nomenclature}

\section{Symbols and abbreviations}

\section{$d \quad$ Size [m]}

$k \quad$ Specific heat ratio

$\dot{m} \quad$ Mass flow rate $[\mathrm{kg} / \mathrm{s}]$

$u \quad$ speed $[\mathrm{m} / \mathrm{s}]$

$C \quad$ Coefficient

$R_{*} \quad$ Specific gas constant [J/kg.K]

$T$ Temperature [K]

$V \quad$ Volume $\left[\mathrm{m}^{3}\right]$

Oh Ohnesorge number $(=\mu / \sqrt{\rho \sigma d})$

Re Reynolds number $(=\rho u d / \mu)$

$W e \quad$ Weber number $\left(=\rho u^{2} d / \sigma\right)$

PSD Particle Size Distribution

SMD Sauter Mean Diameter $[\mu \mathrm{m}]$

$\mu \quad$ Viscosity [Pa s]

$\rho \quad$ Density $\left[\mathrm{kg} / \mathrm{m}^{3}\right]$

$\sigma \quad$ Surface tension $[\mathrm{N} / \mathrm{m}]$

$\tau \quad$ Breakup time constant $\left(=d / u \sqrt{\rho_{l} / \rho_{g}}\right)$
Subscripts

$0 \quad$ Reference state

$b \quad$ Bulk

c Child

d Discharge

fin Final

$g \quad$ Gas

$i \quad$ Inside

isen Isentropic

ini Initiation

$l \quad$ Liquid

$o \quad$ Outside

$r \quad$ Relative

$s \quad$ Surface

\section{References}

[1] Marco, G. I., Vicente, J., and Gaspar, F., 2010, "Scale-up methodology for pharmaceutical spray drying," Chimica Oggi, 28(4), pp. 18-22.

[2] Varga, C. M., Lasheras, J. C., and Hopfinger, E. J., 2003, "Initial breakup of a smalldiameter liquid jet by a high-speed gas stream," Journal of Fluid Mechanics, 497(497), pp. 405-434.

[3] Aliseda, A., Hopfinger, E. J., Lasheras, J. C., Kremer, D. M., Berchielli, A., and Connolly, E. K., 2008, "Atomization of viscous and non-newtonian liquids by a coaxial, high-speed gas jet. Experiments and droplet size modeling," International Journal of Multiphase Flow, 34(2), pp. 161-175.

[4] Joseph, D. D., Belanger, J., and Beavers, G. S., 1999, "Breakup of a liquid drop suddenly exposed to a high-speed airstream," International Journal of Multiphase Flow, 25, pp. 1263-1303.

[5] Joseph, D. D., Beavers, G. S., and Funada, T., 2002, "Rayleigh-Taylor instability of viscoelastic drops at high weber numbers," Journal of Fluid Mechanics, 453, pp. 109-132.

[6] Marmottant, P. and Villermaux, E., 2004, "Fragmentation of stretched liquid ligaments," Physics of Fluids, 16(8), pp. 2732-2741.

[7] Odier, N., Balarac, G., and Corre, C., 2018, "Numerical analysis of the flapping mechanism for a two-phase coaxial jet," International Journal of Multiphase Flow, 106, pp. 164-178.

[8] Tsai, S. C., Ghazimorad, K., and Viers, B., 1991, "Airblast atomization of micronized coal slurries using a twin-fluid jet atomizer," Fuel, 70(4), pp. 483-490.

[9] Jackiw, I. M. and Ashgriz, N., 2021, "On aerodynamic droplet breakup," Journal of Fluid Mechanics, 913(A33).

[10] Igra, D. and Takayama, K., 2001, "Investigation of aerodynamic breakup of a cylindrical water droplet," .

[11] Kumar, A. and Sahu, S., 2020, "Influence of nozzle geometry on primary and large-scale instabilities in coaxial injectors," Chemical Engineering Science, 221, p. 115694.

[12] Hsiang, L. P. and Faeth, G. M., 1992, "Near-limit drop deformation and secondary breakup," International Journal of Multiphase Flow, 18(5), pp. 635-652. 